Hernández-Morales, A., Herrero-Pérezrul, M.D., \& Vázquez-Arce, D.I. (2021). Variabilidad en el tamaño y alimentación de Acanthaster planci (Echinodermata: Asteroidea) en el sur del Golfo de California, México. Revista de Biología Tropical, 69(S1), 185-201. DOI 10.15517/rbt.v69iSuppl.1.46352

DOI 10.15517/rbt.v69iSuppl.1.46352

\title{
Variabilidad en el tamaño y alimentación de Acanthaster planci (Echinodermata: Asteroidea) en el sur del Golfo de California, México
}

\author{
Alejandro Hernández-Morales ${ }^{1 *}$ \\ María Dinorah Herrero-Pérezrul ${ }^{2}$ \\ Daniel Israel Vázquez-Arce ${ }^{3}$
}

1. Laboratorio de Sistemas Arrecifales. Departamento de Biología Marina, Universidad Autónoma de Baja California Sur. Apartado Postal 19-B, C.P. 23080. La Paz, Baja California Sur, México; hdezmorale@gmail.com (*Correspondencia).

2. Centro Interdisciplinario de Ciencias Marinas, Instituto Politécnico Nacional, Ave. Instituto Politécnico Nacional s/n, Col. Playa Palo de Santa Rita, C.P. 23096, La Paz, Baja California Sur, México; dherrero@ipn.mx

3. Centro de Investigación en Maricultura Sustentable, S.C. Fraccionamiento Calle De Los Delfines \#225, Fidepaz, La Paz, C.P. 23090, La Paz, Baja California Sur, México; danielvazarc@gmail.com

Recibido 30-VI-2020. Corregido 07-IX-2020. Aceptado 05-XI-2020.

\author{
ABSTRACT \\ Variability of size and food type of Acanthaster planci (Echinodermata: Asteroidea) \\ in the southern Gulf of California, Mexico
}

\begin{abstract}
Introduction: Little is known about the biology and population dynamics of Acanthaster planci in Mexico. Its size is considered relevant because it is related to consumption rate. Objective: To describe the variability of the disk diameter and food type of the species in four sampling sites in the southern Gulf of California. Methods: Each site was visited on a monthly basis from February 2008 to March 2009. Disk diameter was registered from a total of 389 observed specimens. Measurements included only the main disk from side to side where the arms begin. A flexible tape was used to measure disk diameter. Three size groups were created for easier explanations: small $(<15 \mathrm{~cm})$, medium $(16-25 \mathrm{~cm})$, and large $(>26 \mathrm{~cm})$. From the total, only 298 asteroids were feeding. Preyed organisms were classified in hard corals, other invertebrates, and algae. The Simpson's Diversity Index was used to calculate the probability that any specimen would be feeding on different types of organisms. A permutational analysis of variance was used to detect differences of disk diameter amongst months and study sites. Results: This population of Acanthaster planci had an average disk diameter $=18.23 \pm 0.21$ $\mathrm{cm}$, with a minimum of $7 \mathrm{~cm}$ and maximum of $36 \mathrm{~cm}$. Size structure was unimodal and largely dominated by medium sized specimens, followed by small ones. The larger were less frequent. Disk diameter was statistically higher in February and lower in the last months of the year. San Rafaelito was the site with the largest and Ensenada de Muertos with the smallest organisms. Medium sized individuals were dominant throughout the year and sampling sites. The small sized fed significantly more on Porites panamensis and coralline algae and turf. Larger specimens had the lowest diversity values, more than $90 \%$ of this size group fed on Pocillopora spp. and very few on macroalgae. Medium sized specimens showed the highest diversity, feeding on all the food types, although as expected, many of them were selective to Pocillopora spp. Conclusions: Acanthaster planci can be considered a medium sized species in the study area. There is an evident food selectivity regarding disk diameter size. The three size groups were positively selective to hard corals, but only the small favored Porites panamensis; and together with large individuals, they had the lowest diversity on food types, whereas medium sized asteroids fed on a high diversity of prey.
\end{abstract}

Key words: crown of thorns starfish; food availability; reefs; size structure; Eastern Tropical Pacific. 
La estrella de mar Acanthaster planci se distribuye en zonas arrecifales del IndoPacífico (Vogler, Benzie, Lessios, Barber, \& Wörheide, 2008; Branham, Reed, Bailey, \& Caperon, 1971; De'ath, Fabricius, Sweatman, \& Puotinen, 2012) y en el Pacífico Oriental Tropical (POT). En México, se encuentra en islas oceánicas como Revillagigedo, Clipperton y Marías, y en el Golfo de California incluyendo islas e islotes (Caso, 1962; Dana \& Wolfson, 1970; Barham, Gowdy, \& Wolfson, 1973; Maluf, 1988; Glynn, Veron, \& Wellington, 1996; Cintra-Buenrostro, Reyes-Bonilla, \& Herrero-Pérezrul, 2005; Honey-Escandón, Solís-Marín, \& Laguarda-Figueras, 2008; Herrero-Pérezrul, Rojero-León, \& Reyes-Boni1la, 2010; Luna-Salguero \& Reyes-Bonilla, 2010). La especie ha sido registrada también en Ecuador (Islas Galápagos) (Glynn, 2003), Colombia (Isla de Malpelo e Isla Gorgona) (Narváez \& Zapata, 2010; Zapata, del Mar Palacios, Zambrano, \& Rodríguez-Moreno; 2017), Panamá (Golfo de Chiriquí) (Alvarado, Guzman, \& Breedy, 2012) y Costa Rica (Isla del Coco e Isla del Caño) (Guzmán \& Cortés, 1989; Alvarado et al. 2016).

Los individuos de la especie en el Pacífico mexicano presentan un disco amplio, con varios brazos relativamente cortos y flexibles, suelen tener 9-17 brazos (Caso, 1962). Su forma general es circular y aplanada, la parte dorsal está cubierta de espinas, anchas en la base y afiladas en la punta, siendo de mayor tamaño en los brazos. Acanthaster planci presenta una coloración variada que va de rojo a naranja e incluso marrón y tonos grises. Es común observar en el dorso un patrón en forma de bandas o anillos de distinto grosor y coloración, alternándose bandas oscuras (rojas, naranjas o marrones) con claras (grises) (Fig. 1). Todas las espinas son de color gris.

En general, A. planci se considera un asteroideo de tamaño grande; llega a alcanzar hasta $60 \mathrm{~cm}$ de diámetro de disco en zonas del Indo-Pacífico (Stump, 1996; Engelhardt et al., 2001; Pratchett, 2005; Miller, Jonker, \& Coleman, 2009, Saponari et al., 2018), mientras que los ejemplares en el POT con frecuencia no superan los $20 \mathrm{~cm}$ (Caso, 1961; Glynn, 1982; Reyes-Bonilla \& Calderon-Aguilera, 1999; Zapata et al., 2017).

Keesing y Lucas (1992), De'ath y Moran (1998), Reyes-Bonilla y Calderon-Aguilera (1999) y Kayal, Lenihan, Pau, Penin, y Adjeroud, (2011) mencionan que el diámetro de A. planci está directamente relacionado con el daño potencial que puede causar a las colonias de coral, es decir, estrellas más grandes
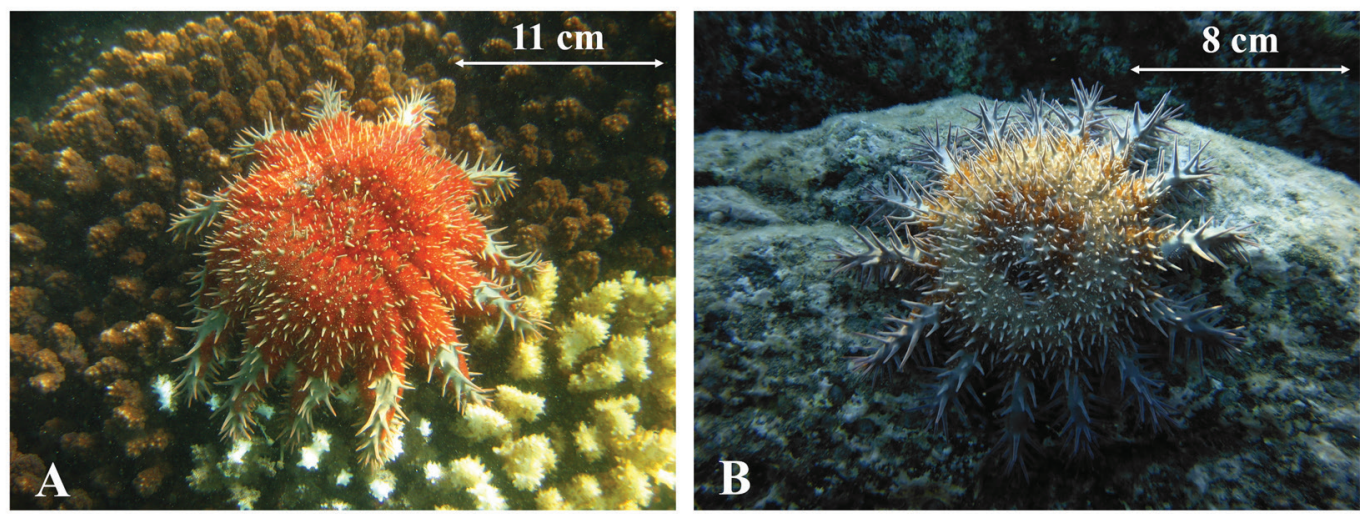

Fig. 1. Ejemplares de Acanthaster planci en comunidades arrecifales de Bahía de La Paz, Baja California Sur, México. A. Sobre coral (Fotografía de Herrero-Pérezrul). B. Sobre tapete de algas (Fotografía de Vázquez-Arce). En la imagen se muestran los patrones de coloración más comunes en el área de estudio.

Fig. 1. Specimens of Acanthaster planci on reef communities from Bahía de La Paz, Baja California Sur, México. A. On coral (Photograph of Herrero-Pérezrul). B. On algal turf (Photograph of Vázquez-Arce). The image shows the most common color patterns observed in the study area. 
consumen más tejido. Adicionalmente, un individuo grande produce millones de huevos más que uno pequeño (Babcock, Milton, \& Pratchett, 2016a). Esta alta fecundidad favorece un crecimiento poblacional acelerado y continuo. Uthicke, Schaffelke, y Byrne (2009) y Kayal et al. (2012), sugieren que abundancias altas promueven el éxito reproductivo provocando los "brotes", que se caracterizan por la presencia de miles de organismos que pueden alcanzar gran tamaño, capaces de ocasionar mortalidad masiva de corales por la gran cantidad de tejido que consumen. De acuerdo con Keesing y Lucas (1992), en la región central de la Gran Barrera Australiana, una estrella con diámetro de $40 \mathrm{~cm}$ puede consumir $478 \mathrm{~cm}^{2}$ de tejido coralino por día durante la temporada cálida, mientras que, en el sur del Golfo de California, una de $21 \mathrm{~cm}$ consume $118.4 \mathrm{~cm}^{2}$ diarios (Reyes-Bonilla \& Calderon-Aguilera, 1999). Estos niveles extrapolados a miles de individuos pueden provocar pérdidas importantes de cobertura coralina, cambios severos en la estructura comunitaria de los arrecifes, incluso de fase (Leray, Béraud, Anker, Chancerelle, \& Mills, 2012; Uthicke et al., 2015; Babcock et al., 2016b), y de manera indirecta pérdidas económicas.

Como los asteroideos, A. planci tiene un estómago flexible que es evertido al exterior. Se denomina comúnmente bolsa gástrica o estomacal. Los pies ambulacrales se adhieren a la presa y ejercen presión desde la parte oral para comenzar la expulsión del estómago, conforme se va desplegando, lo acomodan para que cubra la mayor superficie por todo el contorno, mientras, los brazos se cierran para asegurarla. El estómago es del tamaño del disco y puede extenderse un poco más (Brauer, Jordan, \& Barnes, 1970) pero siempre queda cubierto por los brazos. Una vez colocado en su lugar, el estómago segrega enzimas digestivas con las que comienza a digerir el tejido, que conforme se liquidifica, va siendo absorbido (Mauzey, Birkeland, \& Dayton, 1968). Los restos de alimento que quedan adheridos a los pliegues del estómago, en su mayoría son estructuras rígidas difíciles de digerir, que son descartadas cuando el estómago se retrae al interior del disco.

Por otro lado, se conoce que $A$. planci además de corales, también se alimenta de otros invertebrados e incluso algas (Barnes, 1966; De Alba, 1978; De'ath \& Moran, 1986; Moore, 1990; Chess, Hobson, \& Howard, 1997; De'ath \& Moran, 1998; Kayal et al., 2011). De igual forma, el tamaño está relacionado con la diversidad de presas que consume la especie. Al parecer, los individuos recién metamorfoseados y los más pequeños se alimentan de algas coralinas (Johnson \& Sutton, 1994; Nakamura et al., 2015); conforme incrementan su diámetro consumen corales, otros invertebrados y algas. Los más grandes prefieren corales hermatípicos o formadores de arrecifes (Kayal et al., 2012; Johansson, Francis \& Uthicke, 2016), principalmente los géneros Pocillopora y Acropora (Glynn, 1974; Keesing \& Lucas, 1992).

Es entonces clara la relevancia de conocer el comportamiento del tamaño de este equinodermo y de qué se está alimentando. En México, además de estimaciones de abundancia y registro de su presencia (Dana \& Wolfson, 1970; Luna-Salguero \& Reyes-Bonilla, 2010), se desconoce su biología y aspectos ecológicos. El objetivo de este trabajo es describir la variabilidad en el diámetro del disco de A. planci y de su alimentación en cuatro sitios al sur del Golfo de California.

\section{MATERIALES Y MÉTODOS}

Área de estudio: Se seleccionaron cuatro sitios de muestreo ubicados al sur del Golfo de California, México (Fig. 2) en donde se ha detectado la presencia de $A$. planci.

Isla Gaviota (IG, Fig. 2) se encuentra dentro de la Bahía de La Paz (24¹7'14.91" N $\& 110^{\circ} 20^{\prime} 26.47^{\prime \prime} \mathrm{W}$ ) en una zona protegida. Se localiza muy cerca del puerto Pichilingue, a donde arriban los barcos turísticos, por lo cual es notoria la influencia humana. La parte marina se caracteriza por la presencia de arrecife. Se pueden observar corales, tanto blandos (Pacifigorgia sp., Leptogorgia sp.) como duros (Pocillopora sp., Porites panamensis). Las 


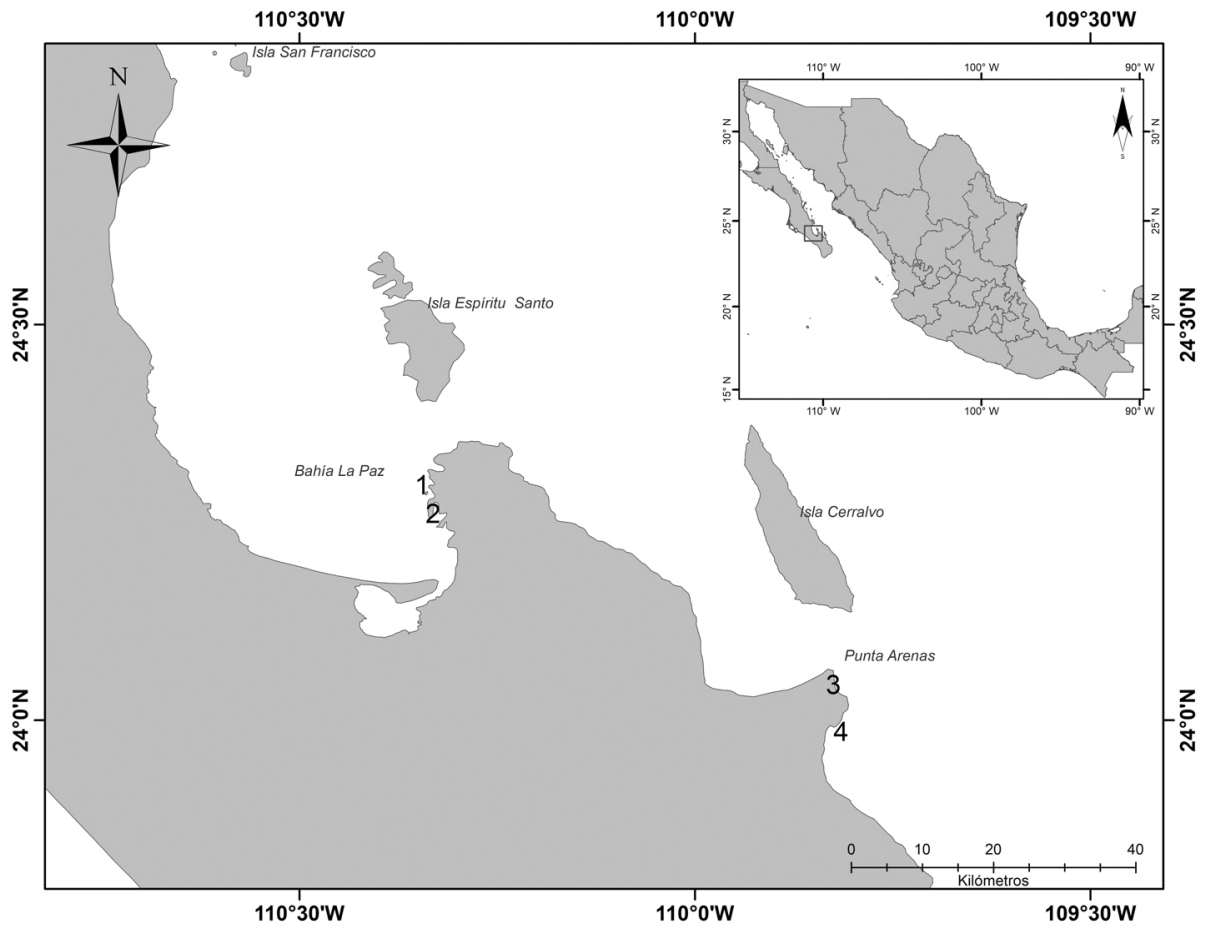

Fig. 2. Ubicación del área de estudio con los sitios de muestreo. 1. San Rafaelito. 2. Isla Gaviota. 3. Cueva de León. 4. Ensenada de Muertos.

Fig. 2. Study area showing sampling sites. 1. San Rafaelito. 2. Isla Gaviota. 3. Cueva de León. 4. Ensenada de Muertos.

algas que dominan son las cafés, pero hay tapetes de algas verdes y algas calcáreas (Carrillo-Domínguez, Casas-Valdez, Ramos-Ramos, Pérez-Gil, \& Sánchez-Rodríguez, 2002). En la parte arenosa, están presentes especies de moluscos, equinodermos y otros invertebrados (Domínguez-Orozco \& Tripp-Quezada, 1997; Solís-Marín, Reyes-Bonilla; Herrero-Pérezrul, Arizpe-Covarrubias, \& Laguarda-Figueras, 1997). El fondo está conformado por corales, roca en forma de cantos rodados y arena. Es un sitio poco profundo, de 0 a $20 \mathrm{~m}$ en la parte que colinda con el canal de San Lorenzo, mientras que la parte cercana al continente está dominada por sustrato arenoso y un máximo de $10 \mathrm{~m}$ de profundidad.

San Rafaelito (SR, Fig. 2), también dentro de la Bahía de la Paz $\left(24^{\circ} 17^{\prime} 52.80^{\prime \prime}\right.$ N \& $110^{\circ} 20^{\prime} 42.00^{\prime}$ ' W), se ubica prácticamente en el canal de San Lorenzo, aproximadamente a dos kilómetros de IG. Es un pequeño islote en donde está colocada una boya de señalamiento marítimo y en las cercanías se ubica una zona de reposo de lobos marinos (Zalophus californianus). El agua de mar es turbia, ya que presenta una gran cantidad de materia orgánica debido a los desechos de comida y heces de estos mamíferos marinos. El fondo está dominado por sustrato coralino, en su mayoría parches de coral del género Pocillopora, también se observa la presencia de cabezas aisladas de P. panamensis y Pavona gigantea, además de algunos corales blandos, varios equinodermos y otros invertebrados. También se encuentra sustrato rocoso y de arena. El arrecife presenta una profundidad máxima de $20 \mathrm{~m}$.

Cueva de León (CL, 24²’34.29" N \& 10949'30.96" W) y Ensenada de Muertos (EM, 2359'29.22” N \& 10949'23.90” W) (Fig. 2) se ubican por fuera de la Bahía de la Paz, hacia el sur, en la parte expuesta al golfo, y son cercanos entre sí. Estos son sitios un 
poco más someros con menos de ocho metros de profundidad; se observan cabezas de coral pequeñas que con frecuencia están alejadas unas de otras y separadas por sustrato rocoso o arenoso. En estos sitios se observa una gran diversidad de algas (tapetes y macroalgas) y corales (Pocillopora spp., P. panamensis, $P$. gigantea y Psammocora sp.) (Reyes-Bonilla \& López-Pérez, 2009).

Trabajo de campo: Los sitios se visitaron mensualmente entre febrero 2008 y marzo 2009, aunque hubo meses en los que no se pudo generar información. El estudio se realizó con tres equipos de dos personas mediante buceo autónomo. La búsqueda de los individuos fue errante y sobre el arrecife; se analizaron todos los organismos encontrados en un periodo de una hora. Durante este trabajo se observó que todos los asteroideos que se estaban alimentando (298 de 389), presentaban el disco con forma abultada evidente, es decir inflada o globosa (Fig. 3A, Fig. 3B, Fig. 3C). Esto se denominó como "postura de alimentación". Los ejemplares en esta postura fueron fotografiados in situ y volteados para identificar el tipo de organismo del que se estaban alimentando a través de la observación de restos presentes en el estómago antes de ser retraído (Fig. 3D). En todos los casos se buscó que el estómago estuviese evertido o fuese evidente, como corroboración de que el animal efectivamente se estaba alimentando. Es importante señalar que, debido al grado de digestión, algunos de los organismos solo pudieron ser identificados a nivel de grandes grupos (e.g. invertebrados y algas). Los corales fueron identificados al nivel taxonómico posible. Una vez registrado el tipo de alimento, los asteroideos fueron colocados sobre el sustrato para que retomaran su forma
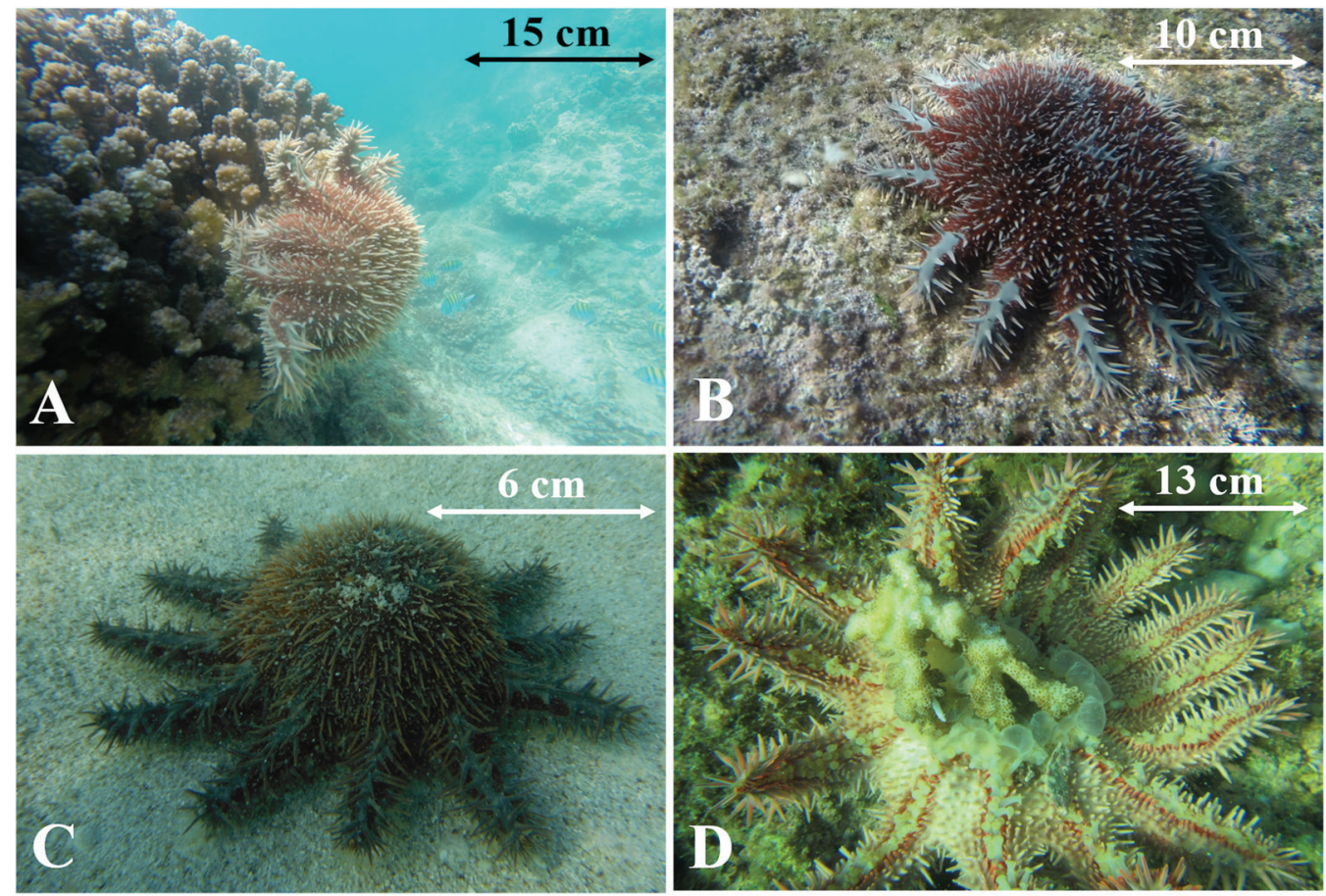

Fig. 3. Ejemplares de Acanthaster planci en postura de alimentación. A. Consumiendo Pocillopora sp. (Fotografía de Hernández-Morales). B. Tapete de algas (Fotografía de Hernández-Morales). C. Invertebrados (Fotografía de VázquezArce). D. Estómago con restos de coral antes de ser retraído (Fotografía cortesía de Paz-García).

Fig. 3. Feeding posture of specimens of Acanthaster planci. A. Eating Pocillopora sp. (Photograph of Hernández-Morales). B. Coral turf (Photograph of Hernández-Morales). C. Invertebrates (Photograph of Vázquez-Arce). D. Stomach folds with coral remains before retracting (Photograph courtesy of Paz-García). 
tradicional, con el cuerpo y brazos extendidos (Fig. 1). Una vez en reposo, se midió el diámetro del disco (Dd) utilizando una cinta métrica flexible (1.0 mm precisión), esta se colocó sobre el disco de lado a lado, justo en el borde donde inician los brazos. Adicionalmente, se registró el tipo de sustrato donde se encontraba el asteroideo, considerando los siguientes tipos: roca, coral y arena.

Análisis de datos: El Dd se agrupó en tres categorías generales, de tal forma que, individuos con diámetro entre 7 y $15 \mathrm{~cm}$ se consideraron pequeños; aquellos de 16 a 25 $\mathrm{cm}$ se consideraron medianos y mayores de 26 $\mathrm{cm}$ se consideraron grandes. Para discernir si individuos del mismo tamaño se alimentan de diferente variedad de organismos, se aplicó el índice de Diversidad de Simpson (1- dominancia) (Krebs, 1999), de acuerdo con la fórmula:

donde: es la proporción del tipo de alimento en el diámetro del asteroideo. Este análisis toma valores de 0 a $1 \mathrm{y}$ permite calcular la probabilidad de que un asteroideo con un Dd dado se esté alimentando de una menor o mayor diversidad de especies (Román-Palacios \& Román-Valencia, 2015).

Se calcularon los estadísticos descriptivos del Dd y para detectar diferencias de esta variable entre los meses de estudio y los sitios de muestreo se aplicó un análisis permutacional de varianza (PERMANOVA) basado en matrices de distancia Euclidiana y 9999 permutaciones
(Palacio, Apodaca, \& Crisci, 2020). Los análisis estadísticos y gráficos se realizaron con el programa $\mathrm{R}$ versión 3.6.3 (R Core Team, 2020) en la plataforma de R Commander (Fox \& Bouchet-Valat, 2020).

\section{RESULTADOS}

Diámetro: Se registró un total de 389 individuos de $A$. planci en los sitios estudio (apéndice 1). La mayoría de los organismos se encontraron sobre sustrato coralino, seguido por rocoso y en tercer lugar sobre arena. Este comportamiento fue similar en los cuatro sitios (Tabla 1). Los asteroideos presentaron un diámetro promedio de $18.23 \pm 0.21 \mathrm{~cm}$, con un mínimo de $7 \mathrm{~cm}$ (EM) y un máximo de 36 cm (SR) (Tabla 1). En general, la distribución del Dd se estructuró de la siguiente manera: el $67.61 \%$ de la población muestreada estuvo representada por individuos medianos ( $\mathrm{Dd}=$ 16 a $25 \mathrm{~cm}$ ), el $27.51 \%$ por pequeños ( $\mathrm{Dd}=$ 7 a $15 \mathrm{~cm})$, el $4.88 \%$ por grandes $(\mathrm{Dd}=26$ a $36 \mathrm{~cm}$ ) y solo se detectó una moda (Fig. 4). El Dd presentó diferencias significativas a lo largo del periodo analizado (Pseudo- $\mathrm{F}_{9,388}=14.106$, $\mathrm{P}=0.0001$ ), donde febrero 2008 (con presencia de individuos grandes), noviembre 2008 y diciembre 2008 (con los más pequeños) fueron los meses que marcaron las diferencias. En la Fig. 5 se presenta la distribución mensual del Dd de la especie en el área de estudio.

Al analizar por sitios, el diámetro de A. planci mostró diferencias significativas

TABLA 1

Número de individuos, diámetro del disco de Acanthaster planci y tipo de sustrato donde se encontraron los ejemplares

TABLE 1

Number of individuals, disk diameter of Acanthaster planci and substrate type were the specimens were found

\begin{tabular}{lccccccc}
\multicolumn{1}{c}{ Sitio } & \multicolumn{3}{c}{ Estimaciones } & \multicolumn{3}{c}{ N por tipo de sustrato } \\
& $\mathrm{N}$ & Media \pm E.S. & Min. & Max. & C & R & A \\
San Rafaelito & 64 & $21.80 \pm 0.52$ & 13 & 36 & 45 & 14 & 5 \\
Isla Gaviota & 115 & $19.03 \pm 0.36$ & 11 & 32 & 79 & 28 & 8 \\
Cueva de León & 116 & $17.69 \pm 0.33$ & 12 & 30 & 102 & 13 & 1 \\
Ensenada de Muertos & 94 & $15.50 \pm 0.34$ & 7 & 24 & 67 & 22 & 5 \\
\hline
\end{tabular}

N: número de individuos; E.S.: error estándar; Min.: mínimo; Max.: máximo; C: coral; R: roca; A: arena. 


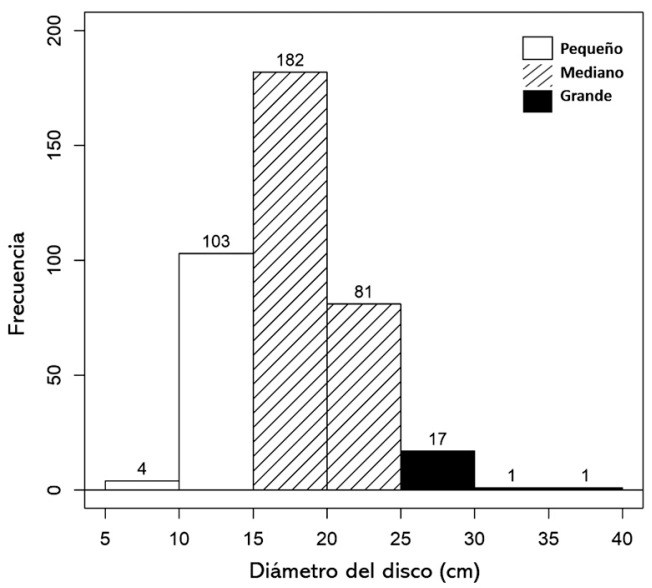

Fig. 4. Distribución de frecuencia de tamaños (diámetro del disco) de Acanthaster planci en el área de estudio.

Fig. 4. Frequency size distribution (disk diameter) of Acanthaster planci at the study site.

(Pseudo- $\left.\mathrm{F}_{3,388}=39.794, \mathrm{P}=0.0001\right)$. En $\mathrm{SR}$ la estructura del Dd fue representada por el $75.01 \%$ de individuos medianos, seguido por los grandes con $15.62 \%$ y los pequeños con $9.37 \%$. Es importante señalar que en este sitio se encontró el individuo con el Dd más grande de todo el periodo analizado $(36 \mathrm{~cm})$ y no se registraron ejemplares con diámetro menor a $10 \mathrm{~cm}$ (Fig. 6A).

Por el contrario, en EM se registraron los individuos más pequeños $(\mathrm{Dd}<10 \mathrm{~cm})$ en toda el área muestreada, representando el $53.18 \%$ del total del sitio, seguidos por los medianos con $46.82 \%$ y no se encontraron ejemplares grandes (Fig. 6B).

Los organismos medianos estuvieron mejor representados en IG con $76.52 \%$ y en $\mathrm{CL}$ con $71.55 \%$, mientras que los grandes tuvieron porcentajes muy bajos de frecuencia, en ambos sitios no superaron el $5 \%$ del total muestreado (Fig. 6C, 6D).

Alimento: Durante el muestreo, no todos los individuos de $A$. planci se estaban alimentando. De los 389 asteroideos registrados en total, solo 298 se encontraron en postura de alimentación, esta fue similar para todos los tipos de alimento (Fig. 3). 279 individuos se encontraron con mayor frecuencia sobre sustrato coralino, 16 sobre rocoso y tres sobre arenoso.

En cuanto a la variedad de alimento, se identificaron ocho tipos de organismos, que se clasificaron en tres grandes grupos: corales, invertebrados y algas. Entre los corales, Pocillopora spp. fue la más depredada con el $51.01 \%$ del total de individuos alimentándose de ella en el área de estudio. El $35.57 \%$ consumió $P$. panamensis, el $7.72 \%$ consumió otras especies de corales (P. gigantea, Psammocora spp.) y el $5.7 \%$ se alimentó de invertebrados y algas (Tabla 2). Los invertebrados estuvieron

TABLA 2

Relación del diámetro del disco con el tipo de organismos de los que se alimentó Acanthaster planci en el área de estudio

TABLE 2

Relationship between disk diameter and type of organisms that Acanthaster planci fed on at the study site

\begin{tabular}{|c|c|c|c|c|}
\hline \multirow{2}{*}{$\begin{array}{l}\text { Grupo de } \\
\text { alimento }\end{array}$} & \multirow{2}{*}{ Tipo de alimento } & \multicolumn{3}{|c|}{ Tamaño de $A$. planci } \\
\hline & & Pequeño \% & Mediano \% & Grande $\%$ \\
\hline \multirow[t]{4}{*}{ Coral duro } & Pocillopora sp..$^{1,2}$ & 32.92 & 55.22 & 93.33 \\
\hline & Porites panamensis ${ }^{1}$ & 52.44 & 31.34 & 0 \\
\hline & Pavona gigantea $^{1}$ & 2.43 & 4.48 & 0 \\
\hline & Psammocora sp. ${ }^{1}$ & 7.30 & 2.99 & 0 \\
\hline \multirow[t]{3}{*}{ Algas } & Alga coralina ${ }^{2}$ & 3.67 & 1.49 & 0 \\
\hline & Macroalga $^{2,3}$ & 0 & 1.49 & 6.67 \\
\hline & Tapete de alga ${ }^{2}$ & 1.24 & 1 & 0 \\
\hline \multirow[t]{2}{*}{ Invertebrados } & Cangrejos, moluscos, opistobranquios y briozoarios ${ }^{2}$ & 0 & 1.99 & 0 \\
\hline & Total & 100 & 100 & 100 \\
\hline
\end{tabular}

1: sustrato coralino; 2 : sustrato rocoso; 3 : sustrato arenoso. 

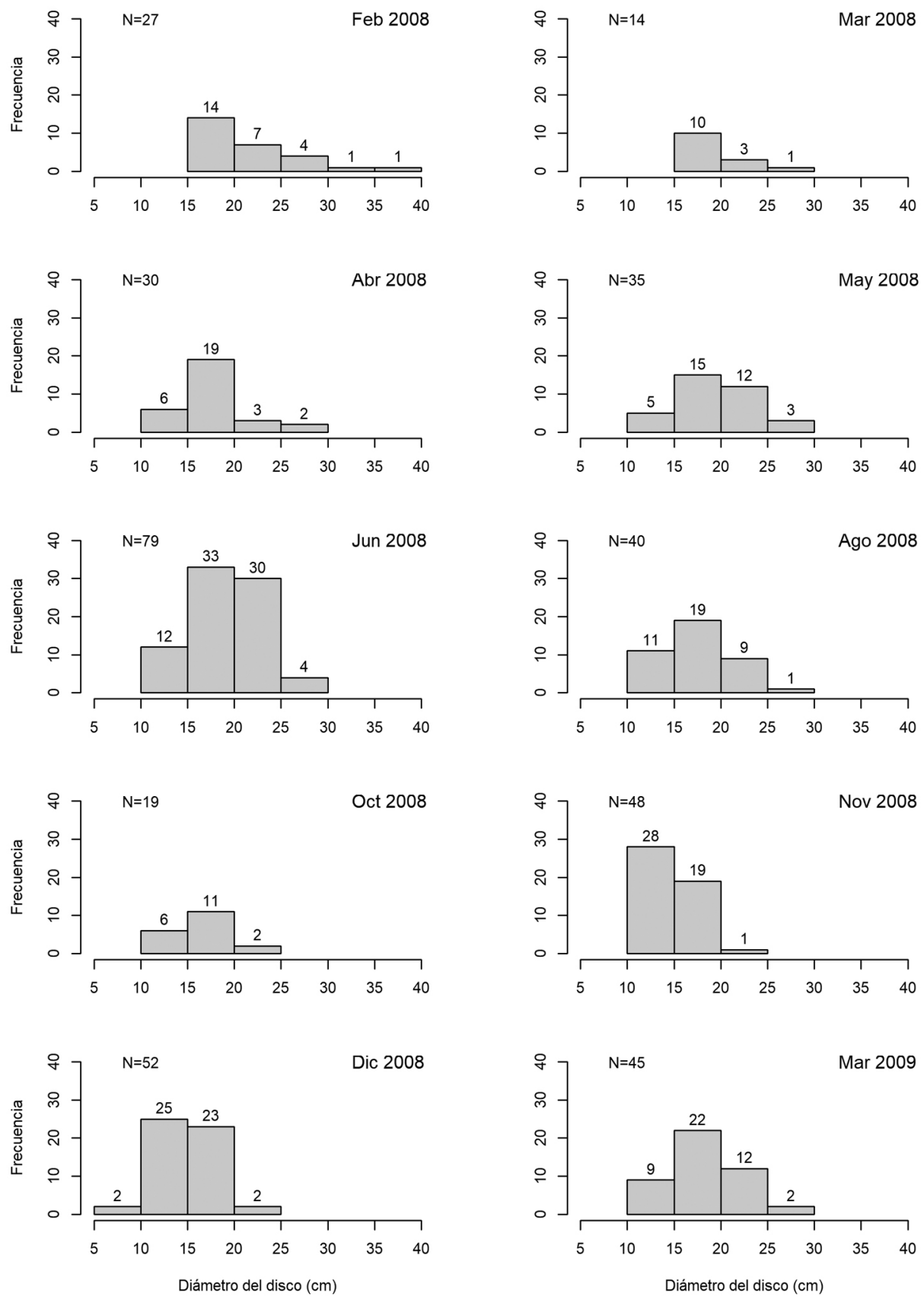

Fig. 5. Distribución mensual del diámetro del disco de Acanthaster planci en el área de estudio. Fig. 5. Monthly distribution of the disk diameter of Acanthaster planci at the study site. 

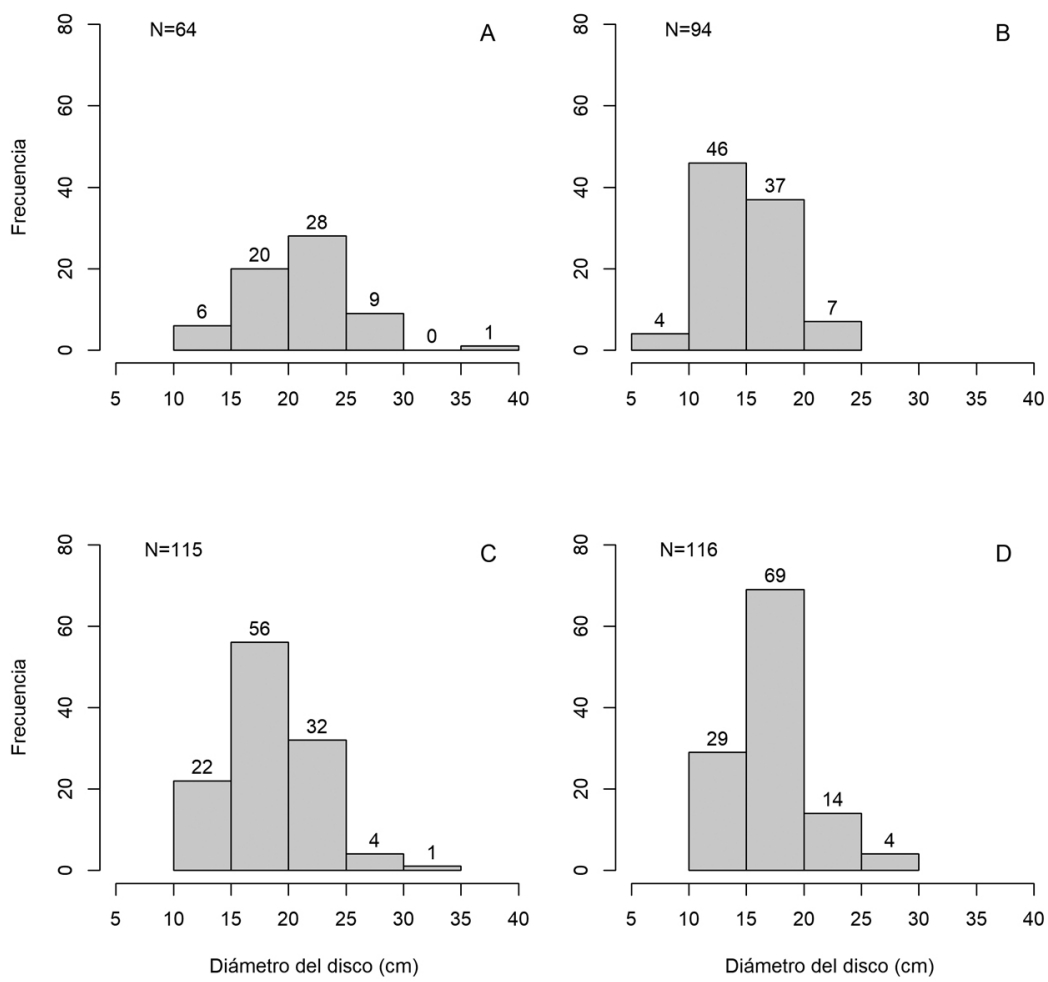

Fig. 6. Distribución de la frecuencia del diámetro del disco de Acanthaster planci en los sitios de muestreo. A. San Rafaelito. B. Ensenada de Muertos. C. Isla Gaviota. D. Cueva de León.

Fig. 6. Disk diameter frequency distribution of Acanthaster planci at sampling sites. A. San Rafaelito. B. Ensenada de Muertos. C. Isla Gaviota. D. Cueva de León.

conformados por crustáceos, moluscos (opistobranquios) y briozoarios, mientras que las algas encontradas fueron coralinas, macroalgas (algas con frondas) y tapetes de algas verdes y cafés.

\section{Diversidad de alimento por grupo de} talla: Se detectó una relación entre la diversidad de alimento y el grupo de talla, donde los individuos medianos se alimentaron de siete de los ocho tipos de alimentos encontrados y presentaron valores altos en el índice de Simpson $(1-\lambda=0.6$ a 0.7$)$ (Fig. 7). El $55.22 \%$ de los asteroideos medianos consumió Pocillopora spp., el $31.34 \%$ P. panamensis y solo el 13.44 $\%$ se alimentó del resto de los grupos. En segundo lugar, el $52.44 \%$ de los organismos pequeños se alimentaron de $P$. panamensis, el $32.92 \%$ de Pocillopora spp. mientras que el
$14.64 \%$ consumió algas, invertebrados y corales duros como Psammocora sp. Los valores del índice para este grupo fueron contrastantes $(1-\lambda=0.0$ a 0.7$)$, lo que sugiere que algunos organismos prefieren una menor diversidad de alimento, mientras que otros del mismo Dd son más variados en su dieta (Fig. 7). En el grupo de los asteroideos grandes, se detectó que la mayoría (93.33\%) consumió únicamente corales del género Pocillopora y apenas el $6.67 \%$ seleccionaron macroalgas (Tabla 2), por esta razón, los valores del índice fueron los más bajos $(1-\lambda=0.0$ a 0.3$)$.

\section{DISCUSIÓN}

Este trabajo es una primera aproximación al entendimiento de la variabilidad del diámetro 


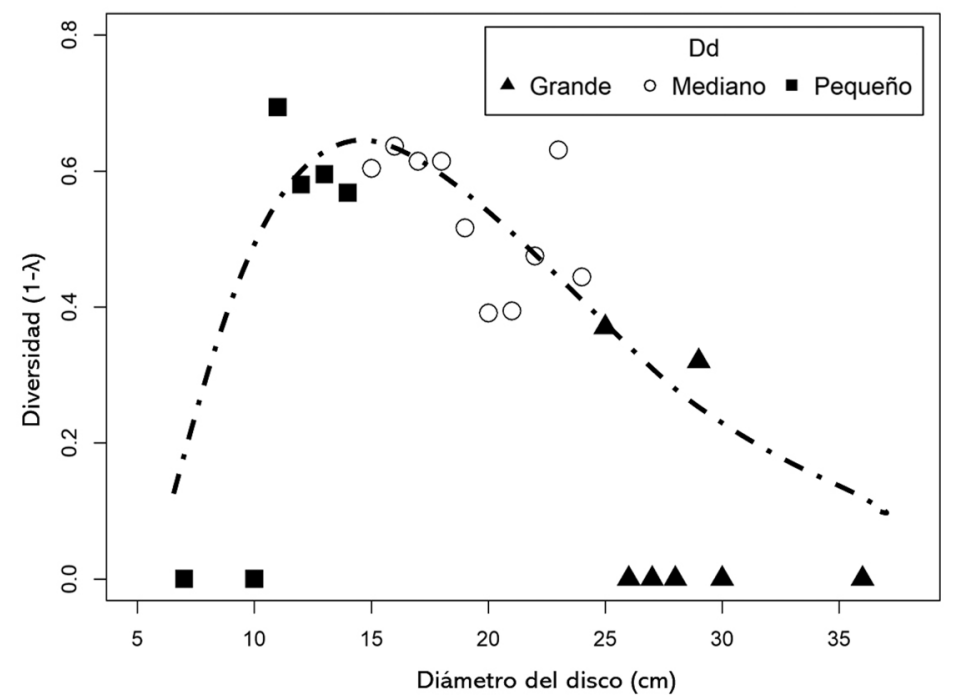

Fig. 7. Diversidad de Simpson de los tipos de alimento con relación al grupo de tamaño de Acanthaster planci. Dd = diámetro del disco.

Fig. 7. Simpson's Diversity of food type according to group size in Acanthaster planci. Dd = disk diameter.

del disco de $A$. planci y de su alimentación en cuatro sitios del sur del Golfo de California. Su tamaño se considera una variable relevante, ya que está relacionado con aspectos como la reproducción (mayor fecundidad) y alimentación, donde organismos grandes consumen más tejido, resultando en una mayor pérdida de cobertura coralina, cambios ecosistémicos y repercusiones en actividades económicas (Keesing \& Lucas, 1992; De'ath \& Moran, 1998; Reyes-Bonilla \& Calderon-Aguilera, 1999; Uthicke et al., 2009; Kayal et al., 2011; 2012; Babcock et al., 2016b).

Diámetro: La distribución del $\operatorname{Dd}$ de $A$. planci en el área de estudio presentó una sola moda y la estructura estuvo conformada en su mayoría por individuos medianos que midieron entre 16 y $25 \mathrm{~cm}$, seguida por los pequeños $(<$ $15 \mathrm{~cm}$ ) y una muy baja proporción de organismos grandes (>26 cm) (Fig. 4); el promedio fue de $18.23 \pm 0.21 \mathrm{~cm}$ con un valor mínimo de $7 \mathrm{~cm}$ y un máximo de $36 \mathrm{~cm}$. Este promedio es mayor al registrado para ejemplares en otras áreas del POT como Panamá $(\mathrm{Dd}=14.6 \mathrm{~cm}$; Glynn, 1982) y Bahía de La Paz (Dd = 10 cm;
Dana \& Wolfson, 1970; Barham et al., 1973). Sin embargo, es mucho menor que el de sus congéneres en el Indo-Pacífico (30-40 cm; Stump, 1996; Pratchett, 2005; Miller, Jonker, \& Coleman, 2009; Saponari et al., 2018). El tamaño de los organismos parece estar relacionado con la cantidad y diversidad de alimento disponible, además del tamaño del área arrecifal (Dana, Newman \& Fager, 1972). Por ejemplo, la Gran Barrera Australiana está dominada por una gran elevada riqueza de especies de coral con coberturas altas (> $60 \%$; Bruno \& Selig, 2007), aportando más comida y espacio para los asteroideos (Reichelt, Bradbury, \& Moran, 1990). Los arrecifes del POT son de tipo rocoso, con coberturas de coral que no superan el $30 \%$ y se distribuyen en parches dominados en su mayoría por un solo género, Pocillopora principalmente, y en menor proporción P. panamensis y P. gigantea (Glynn, 1974; Reyes-Bonilla et al., 2005; Reyes-Bonilla \& López-Pérez, 2009). Esto puede representar una limitante para el tamaño de $A$. planci en esta región.

La distribución del diámetro de $A$. planci se comporta de manera similar tanto en regiones 
del Indo-Pacífico como en las del POT. Las poblaciones se caracterizan por ser unimodales y por la dominancia de individuos con tamaños medianos, seguidos por los pequeños y en menor proporción por los grandes (Dana et al., 1972; Pratchett, 2005). Este patrón solo se invierte durante los eventos plaga o brotes, donde dominan organismos grandes, seguidos por los medianos y en menor proporción por los pequeños (Zann, Brodie, \& Vuki, 1990); lo anterior puede atribuirse a la disponibilidad, tipo de alimento y defensas de la presa.

La poca representatividad de individuos pequeños en la población puede deberse a que ocupan hábitats crípticos que los vuelven difíciles de encontrar. Los individuos grandes fueron poco frecuentes en este trabajo y es interesante mencionar que en otras regiones los individuos más grandes también están poco representados en la población (Dana et al., 1972; Bos, Gumanao, Mueller, \& Saceda-Cardoza, 2013), excepto durante los brotes, como se mencionó anteriormente. Los autores mencionan que siempre están expuestos sobre el sustrato y no se esconden de sus depredadores, por lo que sería de esperarse una frecuencia más elevada. Se desconoce si es debido a que migren a zonas más profundas o posiblemente hayan muerto, ya que no se encuentran en otros lados.

Al analizar por sitio, el Dd de A. planci marcó diferencias significativas debido a los valores extremos, el máximo $(36 \mathrm{~cm})$ se encontró en SR y el mínimo $(7 \mathrm{~cm})$ en EM. Cabe resaltar que, en EM solo se encontraron individuos pequeños y medianos, pero ninguno grande (Tabla 1). En este sitio el coral está distribuido en pequeños parches separados por roca o arena, además incluye cantos rodados y presencia de tapetes de algas; Johnson y Sutton (1994) y Nakamura et al. (2015) sugieren que estos sustratos estimulan y favorecen el establecimiento de las larvas. La heterogeneidad en el sustrato de este sitio parece favorecer la presencia de organismos pequeños, al proveerles refugio más que alimento. Esto se observó durante el presente estudio, ya que los individuos pequeños en su mayoría solo consumieron P. panamensis, seguidas de otros corales duros, pero ningún tipo de algas. A pesar de que en este sitio se encontraron siete de los ocho tipos de organismos de los que se alimenta, la mayoría de los medianos y casi todos los grandes prefirieron Pocillopora spp. (Tabla 2). Los valores máximos de diversidad de Simpson se encontraron en EM (Fig. 7).

En este estudio se detectaron diferencias estadísticas del Dd entre los meses analizados, encontrando a los más grandes en febrero 2008 y los más pequeños hacia el final del año. Parece ser un carácter compartido con otras especies de equinodermos arrecifales en zonas tropicales (Yamaguchi, 1977; HerreroPérezrul, Reyes-Bonilla, García-Domínguez, \& Cintra-Buenrostro, 1999; Reyes-Bonilla \& Herrero-Pérezrul, 2003; Herrera-Escalante, 2005; Díaz-Martínez, Carpizo-Ituarte \& Benítez-Villalobos, 2019).

Algunos autores sugieren que la alta presencia de nutrientes causada por eventos de surgencia y baja temperatura, traen como consecuencia una mayor disponibilidad de alimento y por lo tanto energía acumulada, que favorece el incremento del tamaño y la reproducción (Lucas, 1982; Kettle \& Lucas, 1987). Individuos con diámetro grande tienen el potencial de producir entre 29 y 38 millones de huevos estacionalmente (Babcock et al., 2016a). De acuerdo con Fabricius, Okaji y De'ath (2010), la presencia de clorofila en el agua, así como temperaturas cálidas, son factores que incrementan la probabilidad de supervivencia de las larvas, por lo que se considera que la disponibilidad de nutrientes en el agua es uno de los factores predominantes en el desarrollo de los brotes. Esta alta fecundidad favorece un crecimiento poblacional acelerado y continuo (Uthicke et al., 2009; Kayal et al., 2012). Algunos autores sugieren que las larvas de la especie se reclutan en aguas más profundas, generalmente asociadas con restos de coral muerto, cantos rodados y tapetes de algas coralinas (Kayal et al., 2012), que les dan protección y sirven de alimento para incrementar su tamaño.

En el área de estudio los eventos de surgencia y bajas temperaturas ocurren entre diciembre 
y marzo (Álvarez-Borrego \& Lara-Lara, 1991; Martínez-Flores, Cervantes-Duarte, \& González-Rodríguez, 2006; Espinosa-Carreón \& Valdez-Holguín, 2007; Álvarez-Borrego, 2012; Escalante, Valdez-Holguín, Álvarez-Borrego, \& Lara-Lara, 2013). Esto ayudaría a explicar la presencia de asteroideos de tallas grandes en febrero, que posiblemente han acumulado la energía que utilizarán para mantener la masa corporal (Reyes-Bonilla, Calderón-Aguilera, Galaviz-López, \& Herrero-Pérezrul, 2018). Por otro lado, individuos pequeños que aparecieron a fin de año y que posiblemente se están incorporando a la población (Herrero-Pérezrul \& Chávez, 2005), adquieren la cantidad de nutrientes que necesitan de las algas coralinas y otros organismos y utilizan la energía para incrementar su talla y alcanzar la primera madurez sexual en el menor tiempo posible.

Diversidad de alimento por grupo de talla: En general, se considera que $A$. planci se alimenta preferentemente de corales, con una marcada selectividad por los hermatípicos, en particular los géneros Pocillopora y Acropora (Glynn, 1974; Keesing \& Lucas, 1992; Kayal et al., 2012; Johansson et al., 2016). En este sentido, Keesing (1990) considera que los corales pocilopóridos son de los que poseen mayor cantidad de energía y contenido proteico, mientras que los porítidos presentan los valores más bajos. Esto podría explicar la razón por la cual la mayoría de los asteroideos grandes y medianos analizados en el presente estudio prefirieron al género Pocillopora sobre P. panamensis. A pesar de que esta última especie es más accesible a la depredación, los asteroideos grandes evitan comerlo. Chess et al. (1997) mencionan que este porítido parece no producir compuestos atrayentes y la consideran como "químicamente críptica", ya que los individuos grandes parecen ignorarlas.

Sin embargo, en el área de estudio quedó evidente que los ejemplares medianos y pequeños de $A$. planci fueron los únicos que tuvieron una marcada preferencia por consumir $P$. panamensis. Es posible que su forma masiva ofrezca una superficie más accesible en la que se pueden apoyar para alimentarse. Además, no posee los sistemas de defensa de los pocilopóridos, como las ramas, que reducen la superficie de consumo, la presencia de moco protector y de simbiontes como crustáceos decápodos (Trapezia sp.), que con las tenazas cortan los pies ambulacrales de $A$. planci y ayudan a proteger los pólipos al obligarla a moverse (Keesing, 1990; Chess et al., 1997). Los autores no reportan evidencia que el consumo de $P$. panamensis provoque daño a los asteroideos pequeños por la presencia de compuestos tóxicos.

A pesar de que los individuos pequeños, medianos y grandes de $A$. planci se alimentaron de coral en este estudio, se detectó una relación significativa entre el tamaño y el tipo de organismo del que se estaban alimentando. La mayoría de los asteroideos pequeños se alimentaron con mayor frecuencia de $P$. panamensis y Pocillopora spp., seguido de otros corales, invertebrados y algas (Tabla 2). Esto coincide con lo reportado para la especie en otras regiones (Barnes, 1966; De Alba, 1978; Moore, 1990; Johnson \& Sutton, 1994; Chess, Hobson \& Howard, 1997). Al parecer, individuos recién metamorfoseados y los más pequeños se alimentan de algas coralinas y tapetes; conforme incrementan su diámetro consumen más especies de corales, otros invertebrados y algas. Los más grandes prefieren corales pocilopóridos en su mayoría, posiblemente la energía adquirida les ayuda para mantener su masa y seguir incrementando su tamaño, y también para la reproducción (Keesing, 1990; Chess et al., 1997; Nakamura et al., 2015).

Por otro lado, Keesing y Lucas (1992) también detectaron una relación entre el tamaño de $A$. planci y el tipo de alimento en la Gran Barrera Australiana. Ellos observaron que los individuos consumen más tejido coralino durante la temporada cálida y menor durante el invierno. De acuerdo con los autores, organismos menores de $40 \mathrm{~cm}$ consumieron entre 357 y $478 \mathrm{~cm}^{2}$ de coral por día durante el verano, y solo $161 \mathrm{~cm}^{2}$ en invierno. En el Golfo de California, México, Reyes-Bonilla y CalderonAguilera (1999) estimaron que la especie tiene la capacidad de devorar diariamente $118.4 \mathrm{~cm}^{2}$ 
de coral en Cabo Pulmo, Baja California Sur, entre abril y mayo; el diámetro máximo que registraron fue de $21 \mathrm{~cm}$.

Considerando que durante los brotes se cuantifican miles de organismos y de gran tamaño, la cantidad de tejido coralino consumido es bastante notoria. En los casos más severos, los corales no tienen la capacidad de regenerar el tejido perdido, por lo que mueren. Las estructuras coralinas se mantienen, por lo que el sustrato sigue disponible para otros organismos que rápidamente las colonizan. Sin embargo, los ensambles de especies de corales e invertebrados asociados se ven fuertemente afectados, ya que cambia su estructura y dominancia de especies, con pérdidas de diversidad y cambios de fase que repercuten en el ecosistema y de forma indirecta en el aspecto económico-social (Leray et al., 2012; Uthicke et al., 2015; Babcock et al., 2016b). De acuerdo con Miller (2002), Lamy, Galzin, Kulbicki, Lison de Loma, y Claudet (2015) y MacNeil et al. (2016), cuando se cuentan más de 40 individuos en todo el arrecife y la cobertura coralina se reduce en $30 \%$, puede considerarse el inicio de un brote.

Considerando que $A$. planci en México es una especie más pequeña que sus congéneres en otras regiones como el Indo-Pacífico, el consumo de corales por grupo de tamaño es menor para los arrecifes del sur del Golfo de California. Sin embargo, dada la evidente preferencia por alimentarse de Pocillopora spp. se incrementa el riesgo potencial de ser depredada en el área de estudio, con lo cual se resalta la relevancia de realizar este tipo de trabajos.

Podemos concluir que el diámetro del disco de $A$. planci puede ser un buen indicador para detectar a tiempo y prevenir brotes. Adicionalmente, conocer el tamaño de los individuos facilitaría la toma de decisiones respecto a su manejo o control, es decir, reubicarlas en zonas alejadas o llegar a extremos de erradicación.

Declaración de ética: los autores declaran que todos están de acuerdo con esta publicación y que han hecho aportes que justifican su autoría; que no hay conflicto de interés de ningún tipo; y que han cumplido con todos los requisitos y procedimientos éticos y legales pertinentes. Todas las fuentes de financiamiento se detallan plena y claramente en la sección de agradecimientos. El respectivo documento legal firmado se encuentra en los archivos de la revista.

\section{AGRADECIMIENTOS}

Al Dr. Héctor Reyes Bonilla por datos proporcionados y valiosos consejos. A la Lic. Noemy Meza Zeferino por la elaboración del mapa. A todos los que apoyaron en el muestreo. A los revisores por sus atinadas observaciones.

\section{RESUMEN}

Introducción: Es poco lo que se conoce sobre aspectos biológicos y poblacionales de Acanthaster planci en México. Su tamaño se ha relacionado con la cantidad de alimento que consume, por lo que es relevante conocer esta variable. Objetivo: Describir la variabilidad en el diámetro del disco de la especie y de su alimentación en cuatro sitios al sur del Golfo de California. Métodos: De febrero 2008 a marzo 2009 se visitaron mensualmente los sitios. Se registró el diámetro del disco de un total de 389 individuos observados. El diámetro del disco se midió con una cinta métrica flexible colocada de lado a lado, en el borde donde comienzan los brazos. Para facilitar la descripción, el diámetro del disco se clasificó en tres grupos: pequeños $(<15 \mathrm{~cm})$, medianos $(16-25 \mathrm{~cm})$ y grandes $(>26 \mathrm{~cm})$. Del total, 298 organismos se encontraron alimentándose. Se clasificaron los tipos de organismos consumidos en corales duros, otros invertebrados y algas. Para calcular la probabilidad de que individuos del mismo tamaño se alimenten de diferente variedad de organismos, se aplicó el índice de Diversidad de Simpson. Con el fin de detectar diferencias del diámetro del disco entre los meses de estudio y los sitios de muestreo, se aplicó un análisis permutacional de varianza. Resultados: Acanthaster planci tuvo un promedio de diámetro del disco de $18.23 \pm 0.21 \mathrm{~cm}$, con un mínimo de $7 \mathrm{~cm}$ y un máximo de $36 \mathrm{~cm}$. La estructura de tallas fue unimodal y se caracterizó por la predominancia de individuos medianos, seguida por los pequeños y en baja proporción por los grandes. El diámetro del disco fue estadísticamente más grande en febrero y más pequeño en los últimos meses del año. En San Rafaelito se encontraron los ejemplares más grandes y en Ensenada de Muertos los más pequeños. Los individuos de tamaño mediano fueron más frecuentes a lo largo del año y en todos los sitios. Los individuos pequeños se alimentaron significativamente 
más de Porites panamensis, seguido de algas coralinas y tapetes. Los más grandes tuvieron los valores de diversidad más bajos, más del $90 \%$ de los individuos en este grupo se alimentaron de Pocillopora spp. y algunos de macroalgas. Los medianos presentaron los valores más altos de diversidad y consumieron todos los tipos de alimento encontrados, sin embargo, como era de esperarse, muchos fueron selectivos hacia Pocillopora spp. Conclusiones: Acanthaster planci se considera un individuo de tamaño mediano en el área de estudio. Se detectó una evidente relación entre el diámetro del disco y la diversidad del tipo de alimento. Los tres grupos de tallas fueron positivamente selectivos hacia corales duros; los más pequeños se alimentaron preferentemente de Porites panamensis y en conjunto con los grandes, presentaron valores bajos de diversidad en el tipo de alimento, mientras que los medianos fueron los que consumieron una mayor diversidad de presas.

Palabras clave: estrella corona de espinas; disponibilidad de alimento; arrecifes; estructura de tallas; Pacífico Oriental Tropical.

\section{REFERENCIAS}

Alvarado, J.J., Guzman, H.M., \& Breedy, O. (2012). Distribución y diversidad de equinodermos (Asteroidea, Echinoidea, Holothuroidea) en las islas del Golfo de Chiriquí, Panamá. Revista de Biología Marina y Oceanografia, 47(1), 13-22.

Alvarado, J.J., Beita-Jiménez, A., Mena, S., FernándezGarcía, C., Guzman-Mora, A.G., \& Cortés, J. (2016). Ecosistemas coralinos del Parque Nacional Isla del Coco, Costa Rica: estructura y comparación 1987-2014. Revista de Biología Tropical, 64(1), S153-S175.

Álvarez-Borrego, S., \& Lara-Lara, J.R. (1991). The physical environment and productivity of the Gulf of California. En J.P. Dauphin \& B. Simoneit (Eds.), The Gulf and Peninsular Province of the Californias (pp. 555-567). Oklahoma, E.U.A: The American Association of Petroleum Geologists.

Álvarez-Borrego, S. (2012). Phytoplankton biomass and production in the Gulf of California: a review. Botanica Marina, 55(2), 119-128.

Babcock, R.C., Milton, D.A., \& Pratchett, M.S. (2016a). Relationships between size and reproductive output in the crown-of-thorns starfish. Marine Biology, 163(234), 1-7.

Babcock, R.C., Dambacher, J.M., Morello, E.B., Plagányi, É.E., Hayes, K.R., Sweatman, H.P.A., \& Pratchett, M.S. (2016b). Assessing different causes of crownof-thorns starfish outbreaks and appropriate responses for management on the Great Barrier Reef. PLoS ONE, 11(12), e0169048.
Barham, E.G., Gowdy, R.W., \& Wolfson, F.H. (1973). Acanthaster (Echinodermata, Asteroidea) in Gulf of California. Fishery Bulletin, 71(4), 927-942.

Barnes, J.H. (1966). The crown-of-thorns starfish as a destroyer of coral. Australian Natural History, 15, 257-261.

Bos, A.R., Gumanao, G.S., Mueller, B., \& Saceda-Cardoza, M.M. (2013). Management of crown-of-thorns sea star (Acanthaster planci L.) outbreaks: removal success depends on reef topography and timing within the reproduction cycle. Ocean \& Coastal Management, 71, 116-122.

Branham, J.M., Reed, S.A., Bailey, J.H., \& Caperon, J. (1971). Coral-eating sea stars Acanthaster planci in Hawaii. Science, 172(3988), 1155-1157.

Brauer, R.W., M.J. Jordan, \& Barnes, D.J. (1970). Triggering of the stomach eversion reflex of Acanthaster planci by coral extracts. Nature, 228, 344-346.

Bruno, J.F., \& Selig, E.R. (2007). Regional decline of coral cover in the Indo-Pacific: timing, extent, and subregional comparisons. PLoS ONE, 2(8), e711.

Carrillo-Domínguez, S., Casas-Valdez, M., Ramos-Ramos, F., Pérez-Gil, F., \& Sánchez-Rodríguez, I. (2002). Algas marinas de Baja California Sur, México: Valor nutrimental. Archivos Latinoamericanos de Nutrición, 52(4), 400-405.

Caso, M.E. (1961). Estado actual de los conocimientos acerca de los equinodermos de México (tesis doctoral). Universidad Nacional Autónoma de México, México.

Caso, M.E. (1962). Estudios sobre asteridos de México. Observaciones sobre especies Pacíficas del género Acanthaster y descripción de una subespecie nueva, Acanthaster ellisii pseudoplanci. Anales del Instituto de Biología, Universidad Nacional Autónoma de México, 32(1-2), 313-331.

Chess, J.R., Hobson, E.S., \& Howard, D.F. (1997). Interactions between Acanthaster planci (Echinodermata, Asteroidea) and scleractinian corals at Kona, Hawai'i. Pacific Science, 51(2), 121-133.

Cintra-Buenrostro, C.E., Reyes-Bonilla, H., \& HerreroPérezrul, M.D. (2005). Oceanographic conditions and diversity of sea stars (Echinodermata: Asteroidea) in the Gulf of California, Mexico. Revista de Biología Tropical, 53(3), 245-261.

Dana, T., \& Wolfson, A.A. (1970). Eastern Pacific crownof-thorns starfish populations in the lower Gulf of California. Transactions of the San Diego Society of Natural History, 16(4), 83-90.

Dana, T.F., Newman, W.A., \& Fager, E.W. (1972). Acanthaster aggregations: Interpreted as primarily 
responses to natural phenomena. Pacific Science, 26, 355-372.

De Alba, C. (1978). Predación de coral por la estrella de mar corona de espinas Acanthaster ellisii (Gray) en el área de Bahía de La Paz. México: Memorias I Simposio de Biología Marina, UABCS.

De'ath, G., \& Moran, P.J. (1998). Factors affecting the behaviour of crown-of-thorns starfish (Acanthaster planci L.) on the Great Barrier Reef: 2: Feeding preferences. Journal of Experimental Marine Biology and Ecology, 220(1), 107-126.

De'ath, G., Fabricius, K.E., Sweatman, H., \& Puotinen, M. (2012). The 27-year decline of coral cover on the Great Barrier Reef and its causes. Proceedings of the National Academy of Sciences, 109(44), 17995-17999.

Díaz-Martínez, J.P., Carpizo-Ituarte, E.J., \& Benítez-Villalobos, F. (2019). Reproductive patterns of the black starry sea urchin Arbacia stellata in Punta Banda, Baja California, Mexico. Journal of the Marine Biological Association of the United Kingdom, 99(6), 1379-1391.

Domínguez-Orozco, A.L., \& Tripp-Quezada, A. (1997). Estructura de la comunidad de macroinvertebrados bentónicos de la Caleta de Balandra, Bahía de La Paz, BCS. En R.J. Urbán \& M. Ramírez (Eds.), La Bahía de La Paz, Investigación y Conservación (pp. 119. 127). La Paz: UABCS, CICIMAR, Scripps Institution of Oceanography.

Engelhardt, U., Hartcher, M., Taylor, N., Cruise, J., Engelhardt, D., Russell, M., Stevens, I., Thomas, G., Williams D., \& Wiseman, D. (2001). Crown-ofthorns starfish (Acanthaster planci) in the central Great Barrier Reef region. Results of fine-scale surveys conducted in 1999-2000. Australia: CRC Reef Research Centre Technical Report No. 32.

Escalante, F., Valdez-Holguín, J.E., Álvarez-Borrego, S., \& Lara-Lara, J.R. (2013). Temporal and spatial variation of sea surface temperature, chlorophyll $a$, and primary productivity in the Gulf of California. Ciencias Marinas, 39(2), 203-215.

Espinosa-Carreón, T.L., \& Valdez-Holguín, E. (2007). Variabilidad interanual de clorofila en el Golfo de California. Ecología Aplicada, 6(1-2), 83-92.

Fabricius, K.E., Okaji, K., \& De'ath, G. (2010). Three lines of evidence to link outbreaks of the crown-of-thorns seastar Acanthaster planci to the release of larval food limitation. Coral Reefs, 29(3), 593-605.

Fox, J., \& Bouchet-Valat, M. (2020). Rcmdr: R Commander. $R$ package version 2.6-2. Recuperado de https:// cran.r-project.org/web/packages/Rcmdr/index.html.
Glynn, P.W. (1974). The impact of Acanthaster on corals and coral reefs in the eastern Pacific. Environmental Conservation, 1(4), 295-304.

Glynn, P.W. (1982). Individual recognition and phenotypic variability in Acanthaster planci (Echinodermata: Asteroidea). Coral Reefs, 1(2), 89-94.

Glynn, P.W., Veron, J.E.N., \& Wellington, G.M. (1996). Clipperton Atoll (eastern Pacific): oceanography, geomorphology, reef-building coral ecology and biogeography. Coral Reefs, 15(2), 71-99.

Glynn, P.W. (2003). Coral communities and coral reefs of Ecuador. En J. Cortés (Ed.). Latin American coral reefs (pp. 449-472). Países Bajos: Elsevier Science.

Guzmán, H.M., \& Cortés, J. (1989). Coral reef community structure at Caño Island, Pacific Costa Rica. Marine Ecology, 10(1), 23-41.

Herrera-Escalante, T. (2005). Dinámica poblacional y reproducción de la estrella de mar Phataria unifascialis (Gray 1840) (Echinodermata: Asteroidea) en Pichilingue, Bahía de La Paz, Baja California Sur, México (Tesis de Maestría). Centro Interdisciplinario de Ciencias Marinas del Instituto Politécnico Nacional, México.

Herrero-Pérezrul, M.D., Reyes-Bonilla, H., GarcíaDomínguez, F., \& Cintra-Buenrostro, C.E. (1999). Reproduction and growth of Isostichopus fuscus (Echinodermata: Holothuroidea) in the southern Gulf of California, Mexico. Marine Biology, 135, 521-532.

Herrero-Pérezrul, M.D., \& Chávez, E.A. (2005). Optimum fishing strategies for Isostichopus fuscus (Echinodermata: Holothuroidea) in the Gulf of California, México. Revista de Biología Tropical, 53(3), 357-366.

Herrero-Pérezrul M.D., Rojero-León S., \& Reyes-Bonilla, H. (2010) Community structure of conspicuous echinoderms of three islands from the Gulf of California, Mexico. En L.G. Harris, S.A. Böttger, C.W. Walker, \& M.P. Lesser (Eds.), Echinoderms: Durham. Proceedings of the 12th International Echinoderm Conference (pp. 615-620). Leiden: CRC Press, Taylor and Francis group, Balkema.

Honey-Escandón, M., Solís-Marín, F.A., \& LaguardaFigueras, A. (2008). Equinodermos (Echinodermata) del Pacífico Mexicano. Revista de Biología Tropical, $56(3), 57-73$

Johnson, C.R., \& Sutton, D.C. (1994). Bacteria on the surface of crustose coralline algae induce metamorphosis of the crown-of-thorns starfish Acanthaster planci. Marine Biology, 120(2), 305-310.

Johansson, C.L., Francis, D.S., \& Uthicke, S. (2016). Food preferences of juvenile corallivorous crownof-thorns (Acanthaster planci) sea stars. Marine Biology, 163, 49. 
Kayal, M., Lenihan, H.S., Pau, C., Penin, L., \& Adjeroud, M. (2011). Associational refuges among corals mediate impacts of a crown-of-thorns starfish Acanthaster planci outbreak. Indirect positive interactions in communities. Coral Reefs, 30(3), 827-837.

Kayal, M., Vercelloni, J., Lison de Loma, T., Bosserelle, P., Chancerelle, Y., Geoffroy, S., Stievenart, C., Michonneau, F., Penin, L., Planes, S., \& Adjeroud, M. (2012). Predator crown-of-thorns starfish (Acanthaster planci) outbreak, mass mortality of corals, and cascading effects on reef fish and benthic communities. PLoS ONE, 7(10), e47363.

Keesing, J.K. (1990). Feeding biology of the crown-ofthorns starfish, Acanthaster planci (Linnaeus) (Tesis de Doctorado). James Cook University, Australia

Keesing, J.K., \& Lucas, J.S. (1992). Field measurement of feeding and movement rates of the crown-of-thorns starfish Acanthaster planci (L.). Journal of Experimental Marine Biology and Ecology, 156(1), 89-104.

Kettle, B.T., \& Lucas, J.S. (1987). Biometric relationships between organ indices, fecundity, oxygen consumption and body size in Acanthaster planci (L.) (Echinodermata; Asteroidea). Bulletin of Marine Science, 41(2), 541-551.

Krebs, C.J. (1999). Ecological Methodology (2a Ed.). California, E.U.A.: Addison Wesley Longman, Menlo Park.

Lamy, T., Galzin, R., Kulbicki, M., Lison de Loma, T., \& Claudet, J. (2016). Three decades of recurrent declines and recoveries in corals belie ongoing change in fish assemblages. Coral Reefs, 35(1), 293-302.

Leray, M., Béraud, M., Anker, A., Chancerelle, Y., \& Mills, S.C. (2012). Acanthaster planci outbreak: decline in coral health, coral size structure modification and consequences for obligate decapod assemblages. PLoS ONE, 7(4), e35456.

Lucas, J.S. (1982). Quantitative studies of feeding and nutrition during larval development of the coral reef asteroid Acanthaster planci (L.). Journal of Experimental Marine Biology and Ecology, 65(2), 173-193.

Luna-Salguero, B.M., \& Reyes-Bonilla, H. (2010). Community and trophic structure of sea stars (Echinodermata: Asteroidea) in rocky reefs of Loreto, Gulf of California, Mexico. Hidrobiológica, 20(2), 127-134.

MacNeil, M.A., Mellin, C., Pratchett, M.S., Hoey, J., Anthony, K.R., Cheal, A.J., Miller, I., Sweatman, H., Cowan, Z.L., Taylor, S., Moon, S., \& Fonnesbeck, C.J. (2016). Joint estimation of crown of thorns (Acanthaster planci) densities on the Great Barrier Reef. PeerJ, 4, e2310.

Maluf, L.Y. (1988). Composition and distribution of the central Eastern Pacific Echinoderms. Natural History
Museum of Los Angeles County, Technical Reports, 2, 1-242.

Martínez-Flores, G., Cervantes-Duarte, R., \& GonzálezRodríguez, E. (2006). Análisis de temperatura superficial del mar en la Bahía de La Paz, Baja California Sur. Naturaleza y Desarrollo, 4(2), 26-34.

Mauzey, K., Birkland, C. \& Dayton, P. (1968). Feeding behavior of asteroids and escape responses of their prey in the Puget Sound region. Ecology, 49(4), 603-619.

Miller, I. (2002). Historical patterns and current trends in the broadscale distribution of crown-of-thorns starfish in the northern and central sections of the Great Barrier Reef. Proceedings of the $9^{\text {th }}$ International Coral Reef Symposium, 2, 1273-1279.

Miller, I.R., Jonker M., \& Coleman, G. (2009). Crown-ofthorns starfish and coral surveys using the manta tow and SCUBA search techniques. Townsville, Australia: Australian Institute of Marine Science.

Moore, R.J. (1990). Persistent and transient populations of the crown-of-thorns starfish, Acanthaster planci. En R.H. Bradbury (Ed.), Acanthaster and the Coral Reef: A theoretical perspective (pp. 236-277). Heidelberg: Springer.

Nakamura, M., Kumagai, N.H., Sakai, K., Okaji, K., Ogasawara, K., \& Mitarai, S. (2015). Spatial variability in recruitment of acroporid corals and predatory starfish along the Onna coast, Okinawa, Japan. Marine Ecology Progress Series, 540, 1-12.

Narváez, K., \& Zapata, F.A. (2010). First record and impact of the crown-of-thorns starfish, Acanthaster planci (Spinulosida: Acanthasteridae) on corals of Malpelo Island, Colombian Pacific. Revista de Biología Tropical, 58(1), 139-143.

Palacio, F.X, Apodaca, M.J., \& Crisci, J.V. (2020). Análisis Multivariado para datos biológicos: Teoría y su aplicación utilizando el lenguaje R. Buenos Aires, Argentina: Fundación de Historia Natural Félix de Azara.

Pratchett, M.S. (2005). Dynamics of an outbreak population of Acanthaster planci at Lizard Island, northern Great Barrier Reef (1995-1999). Coral Reefs, 24(3), 453-462.

R Core Team. (2020). R: A language and environment for statistical computing. R Foundation for Statistical Computing. Vienna, Austria. Recuperado de https:// www.R-project.org/

Reichelt, R.E., Bradbury, R.H., \& Moran, P.J. (1990). The crown-of-thorns starfish, Acanthaster planci, on the Great Barrier Reef. Mathematical and Computer Modelling, 13(6), 45-60.

Reyes-Bonilla, H., \& Calderon-Aguilera, L.E. (1999). Population density, distribution and consumption 
rates of three corallivores at Cabo Pulmo Reef, Gulf of California, Mexico. Marine Ecology, 20(3-4), 347-357.

Reyes-Bonilla, H., \& Herrero-Pérezrul, M.D. (2003). Population parameters of an exploited population of Isostichopus fuscus (Holothuroidea) in the southern Gulf of California, Mexico. Fisheries Research, $59(3), 423-430$.

Reyes-Bonilla, H., Calderón-Aguilera, L.E., Cruz-Piñón, G., Medina-Rosas, P., López-Pérez, R.A., Herrero Pérezrul, M.D., Leyte Morales, G.E., Cupul-Magaña, A.L., \& Carriquiry-Beltrán, J.D. (2005). Atlas de corales del Pacífico Mexicano. México: CICESE, CONABIO, CONACyT, UABCS, UDG, UMAR.

Reyes-Bonilla, H., \& López-Pérez, R.A. (2009). Corals and coral reef communities in the Gulf of California. En A. Johnson \& J. Ledezma-Vázquez (Eds.), Atlas of coastal ecosystems in the western Gulf of California (pp. 45-57). Tucson: The University of Arizona Press.

Reyes-Bonilla, H., Calderón-Aguilera, L.E., GalavizLópez, J.M., \& Herrero-Pérezrul, M.D. (2018). Size and relative condition index of the brown sea cucumber Isostichopus fuscus in the northern Gulf of California, Mexico. Latin American Journal of Aquatic Research, 46(4), 831-836.

Román-Palacios, C., \& Román-Valencia, C. (2015). Hábitos tróficos de dos especies sintópicas de carácidos en una quebrada de alta montaña en los Andes colombianos. Revista Mexicana de Biodiversidad, 86(3), 782-788.

Saponari, L., Montalbetti, E., Galli, P., Strona, G., Seveso, D., Dehnert, I., \& Montano, S. (2018). Monitoring and assessing a 2-year outbreak of the corallivorous seastar Acanthaster planci in Ari Atoll, Republic of Maldives. Environmental Monitoring and Assessment, 190(6), 344
Solís-Marín, F.A., Reyes-Bonilla, H., Herrero-Pérezrul, M.D., Arizpe-Covarrubias, O., \& Laguarda-Figueras, A. (1997). Systematics and distribution of the echinoderms from Bahía de la Paz. Ciencias Marinas, 23(2), 249-263.

Stump, R. (1996). An investigation to describe the population dynamics of Acanthaster planci (L.) around Lizard Island, Cairns section, Great Barrier Reef Marine Park (No. 10). Australia: CRC Reef Research Centre.

Uthicke, S., Schaffelke B., \& Byrne, M. (2009). A boombust phylum? Ecological and evolutionary consequences of density variations in echinoderms. Ecological Monographs, 79(1), 3-24.

Uthicke, S., Logan, M., Liddy, M., Francis, D., Hardy, N., \& Lamare, M. (2015). Climate change as an unexpected co-factor promoting coral eating seastar (Acanthaster planci) outbreaks. Scientific Reports, 5,8402 .

Vogler, C., Benzie, J., Lessios, H., Barber, P., \& Wörheide, G. (2008). A threat to coral reefs multiplied? Four species of crown-of-thorns starfish. Biology Letters, 4(6), 696-699.

Yamaguchi, M. (1977). Population structure, spawning, and growth of the coral reef asteroid Linckia laevigata (Linnaeus). Pacific Science, 31(1), 13-30.

Zann, L., Brodie, J., \& Vuki, V. (1990). History and dynamics of the crown-of-thorns starfish Acanthaster planci (L.) in the Suva area, Fiji. Coral Reefs, 9(3), 135-144.

Zapata, F.A., del Mar Palacios, M., Zambrano, V., \& Rodríguez-Moreno, M. (2017). Filling the gaps: first record of the crown-of-thorns starfish, Acanthaster planci (Linnaeus, 1758) (Spinulosida: Acanthasteridae), at Gorgona Island, Colombia, Tropical Eastern Pacific. Check List, 13(3), 2112.

See Digital Appendix at: / Ver Apéndice digital en: revistas.ucr.ac.cr 